\title{
Characterisation of the unstable expanded CAG repeat in the MJD1 gene in four Brazilian families of Portuguese descent with Machado-Joseph disease
}

Giovanni Stevanin, Eloy Cassa, Géraldine Cancel, Nacer Abbas, Alexandra Dürr, Edymar Jardim, Yves Agid, Patricia S Sousa, Alexis Brice

\section{INSERM U289, Hôpital de la which suggests the existence of a common founder.} Salpêtrière, 47 Bd de l'Hôpital, 75651 Paris Cédex 13, France

G Stevanin

G Cancel

A Dürr

Y Agid

A Brice

Departamento de Neurologia,

Hospital das Clinicas, Universidade de Sao

Paulo, Campus de

Monte Alegre,

Ribeirao Preto,

Sao Paulo,

Brazil

E Cassa

E Jardim

P S Sousa*

*Present address: Departamento de

Neurologia de Faculdade

de Medicina, Universidade de Sao Paulo, Sao Paulo,

Sao Paulo,
Brazil.

Correspondence to:

Dr Brice

Received 28 March 1995 Revised version accepted for publication 15 May 1995

( $\mathcal{F}$ Med Genet 1995;32:827-830)

Machado-Joseph disease (MJD) is an autosomal dominant neurological disorder characterised by cerebellar ataxia variably associated with pyramidal signs, a dystonicrigid extrapyramidal syndrome, amyotrophy, facial-lingual fasciculations, and progressive external ophthalmoplegia. ${ }^{12}$ Originally described in Azorean families of Portuguese descent, this disease occurs in other ethnic groups as well. ${ }^{3-5}$ Because of clinical and genetic similarities to autosomal dominant cerebellar ataxia (ADCA) type I, MJD has been classified as a form of ADCA type $\mathrm{I}^{6}{ }^{6}$

In Japanese kindreds, Takiyama et $a l^{7}$ assigned the MJD gene to chromosome 14q24.3q 32 , in a region of approximately $29 \mathrm{cM}$, which was later reduced to $18^{8}$ then 3 to $4 \mathrm{cM}^{910}$ in Portuguese-Azorean, Brazilian, and Japanese families, respectively. Recently, the MJD gene was identified and found to contain an unstable CAG repeat that is expanded in affected subjects. ${ }^{11}$ This gene is identical to the spinal cerebellar ataxia 3 (SCA3) gene since it has been

\begin{abstract}
Machado-Joseph disease (MJD) is an autosomal dominant neurodegenerative disorder which has been shown to result, in Japanese families, from the expansion of a CAG repeat in the MJD1 gene on chromosome 14q. We show that the same molecular mechanism is responsible for MJD in four large Brazilian kindreds of Portuguese descent. The behaviour of the mutation was evaluated in 28 affected and 19 asymptomatic gene carriers. The number of repeats in the expanded alleles ranged from 66 to 77 with a strong negative correlation with age at onset $(r=0 \cdot 79)$. A mean 1.6 repeats increase from generation to generation correlated with clinical anticipation. Instability of the CAG repeat was bidirectional, with expansions as well as contractions, and was more marked in paternal transmissions. Finally, linkage disequilibrium was complete at locus D14S280 in the four Portuguese-Brazilian kindreds and four previously reported French families with the same mutation,
\end{abstract}

mapped to the same $3 \mathrm{cM}$ on chromosome $14 \mathrm{q}^{12}$ and expanded alleles in the MJD1 gene are observed in all SCA3 patients. ${ }^{12 \mathrm{~A}}$

We have now collected and analysed four Brazilian kindreds of Portuguese descent presenting with MJD, in one of which (GC1) linkage to the MJD locus has previously been reported. ${ }^{9}$ Expansion of the CAG repeat at the SCA3/MJD locus is present in all patients. The behaviour of the mutation has been analysed.

\section{Materials and methods}

SUBJECTS

One hundred and four members from four Brazilian families (GC1, GC2, GC4, and GC5), including 28 patients, were examined and blood was taken for DNA extraction. All patients fulfilled the diagnostic criteria of MJD. ${ }^{12}$ A detailed description of these kindreds has been reported elsewhere (Cassa et al, in preparation). Although the same frequent Portuguese surname was found among the affected ancestors of the four kindreds, the families, whose genealogies were traced back to 1820 , did not appear to be related.

Statistical analyses were performed using the non-parametric, Mann-Whitney U, and Kruskall-Wallis tests for comparison of means, the $F$ test for comparison of variances and the Yates Corrected $\chi^{2}$ for comparison of percentages and linkage disequilibrium. The age at onset of 16 dead patients was included in the anticipation calculation.

\section{GENOTYPING}

Genotypes at the MJD locus (MJD1 gene) were determined by PCR amplification as previously described, ${ }^{11}$ except that an annealing temperature of $55^{\circ} \mathrm{C}$ was used. Four microsatellites from the SCA3/MJD region (D14S291, D14S280, AFM343vfl, and D14S81) were also amplified by PCR as previously described. ${ }^{12}$

Aliquots of the PCR products were then diluted 1:2 in formamide loading buffer, denatured for 10 minutes at $94^{\circ} \mathrm{C}$, and run on a $6 \%$ acrylamide $/ 7 \mathrm{~mol} / \mathrm{l}$ urea gel. After blotting on nylon membrane (Hybond $\mathrm{N}+$, Amersham), hybridisation was carried out as described previously ${ }^{12}$ with $5^{\prime} \gamma \mathrm{P}^{32}$ labelled $(\mathrm{CA})_{10}$ and $(\mathrm{CAG})_{7}$ oligonucleotides. Membranes were washed in $2 \times \mathrm{SSC}, 0.1 \%$ SDS for 10 minutes at room temperature and exposed for 


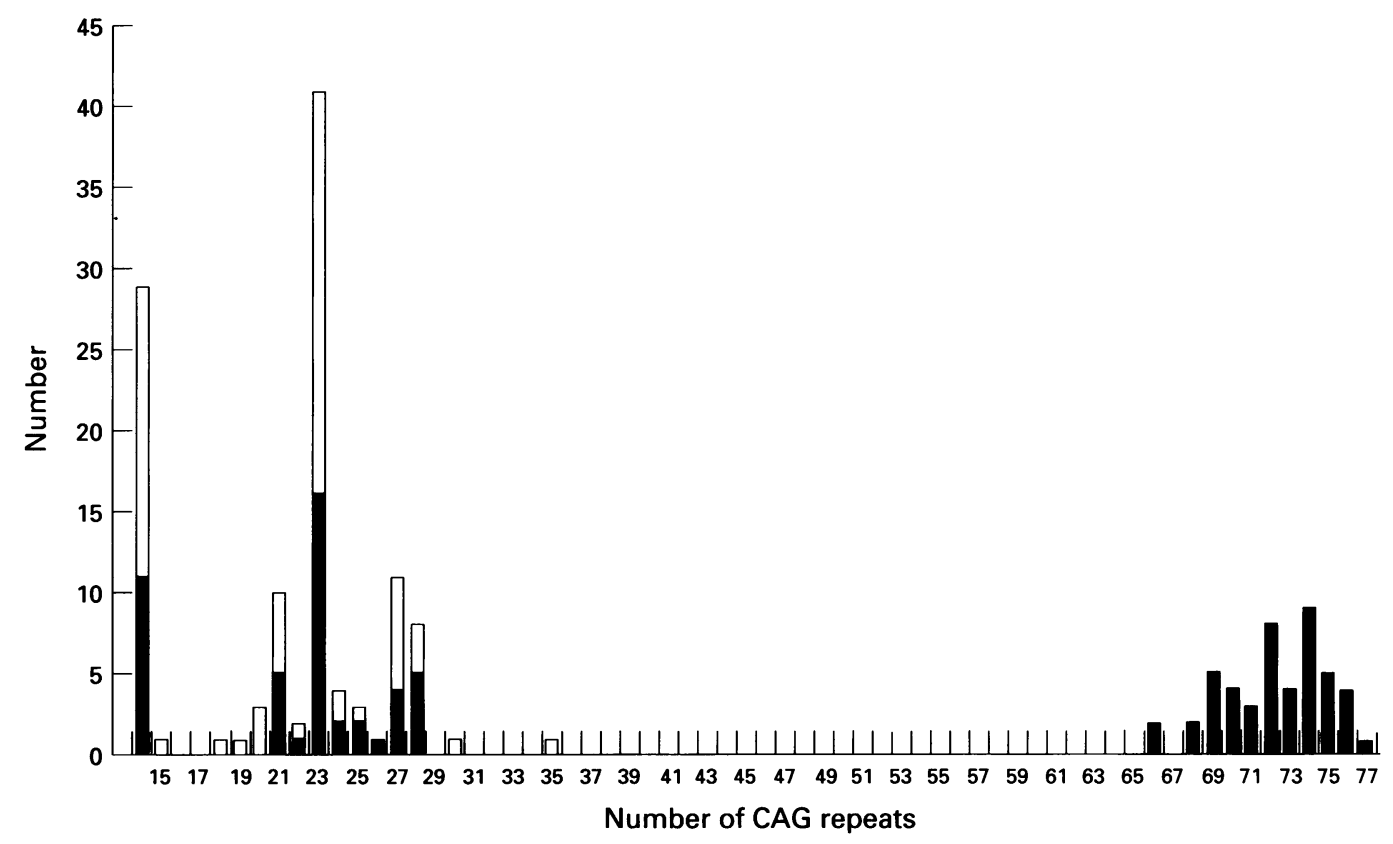

Figure 1 Distribution of the number of CAG repeats in normal $(n=108)$ and expanded $(n=47)$ alleles. White bars $=$ controls, black bars = patients.

two to eight hours to $x$ ray film for autoradiography. Allele sizes were determined by comparison with an M13 sequencing ladder. The allele numbering for the microsatellite markers was as in Stevanin et al. ${ }^{12}$

\section{Results}

MOLECULAR CHARACTERISATION OF THE MUTATION

All patients $(n=28)$ from the four Brazilian families were heterozygous for the mutation, with a number of CAG repeats in the normal range (14 to 28 ) in one allele, but increased by at least 38 repeats (range 66 to 77 ) in the other. Nineteen out of 58 at risk subjects also carried an allele with an expanded CAG repeat. Analysis of 70 normal chromosomes from 35 unrelated Brazilian controls and of 48 normal chromosomes from the patients and at risk carriers showed the existence of 15 different alleles ranging from 14 to 35 CAG repeats. Their distribution was not significantly different from that observed in Japanese ${ }^{13}$ and French controls. ${ }^{12 \mathrm{~A}}$ Two major alleles, containing 14 and 23 repeats with a frequency of

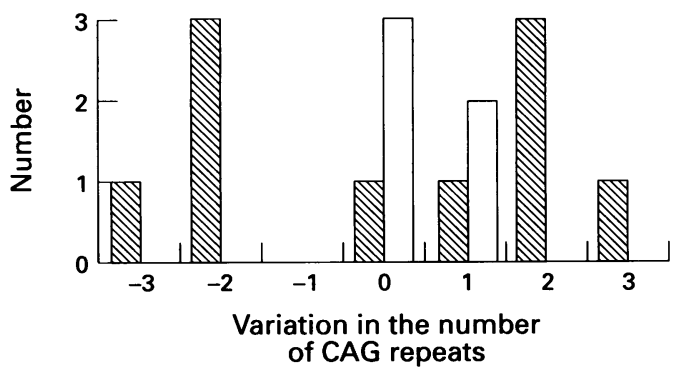

Figure 2 Variation of the number of repeats in the expanded allele in 15 parent-child couples. Paternal and maternal transmissions are represented by hatched and white bars, respectively.
$23 \%$ and $35 \%$, respectively, were detected. As shown in fig 1 , there was a clear cut difference between the normal and expanded alleles.

\section{BEHAVIOUR OF THE MUTATION}

The normal allele was always transmitted with the same number of repeats. The size of the pathological allele varied in most of the transmissions (fig 2) with a tendency to increase in successive generations (table). The mean increase per generation was 1.6 repeats when generation III, in which only one person was tested, was excluded $(p<0.05)$.

Repeat size increased in seven and decreased in four out of 15 parent-child pairs, resulting in a mean increase of +0.2 repeat. Mean alteration in repeat size was similar whether maternally $(+0 \cdot 4, n=5)$ or paternally $(+0 \cdot 1, n=$ 10) transmitted (fig 2). However, the range of variation (fig 2) was larger in paternal (variance $=4.7$ ) than in maternal (variance $=0.3$ ) transmissions $(p<0.05)$. Furthermore, analysis of variance of 11 children from five sibships who received the mutation from their mother compared to 21 children from 10 sibships who received the mutated allele from their father, showed a greater variance when the disease was paternally $(3 \cdot 1)$ than maternally $(0 \cdot 3)$ transmitted $(\mathrm{p}<0.05)$.

The mean number of CAG repeats was similar in men $(n=26,72.7$ (SD 3.0) repeats) and women $(n=21,71 \cdot 7$ (SD 2.3) repeats).

\section{CLINICAL CORRELATIONS}

The mean age at onset was 45 (SD13) (range 25-72) years and decreased in successive generations: GII, $72(n=1)$; GIII, 53 (SD12) $(n=$ 10); GIV, 47 (SD 12) $(n=25)$, and GV, 32 (SD 5) $(n=10)(p<0 \cdot 01)$. In the 22 parentchild pairs for which the ages at onset were 
Number of $C A G$ repeats in the expanded allele in successive generations

\begin{tabular}{llc}
\hline Generation & No of CAG repeats (range) & No of subjects \\
\hline III & 66 & 1 \\
IV & $71 \cdot 2$ (SD 2.7) & 18 \\
V & $(66-76)$ & \\
& $73 \cdot 0$ (SD 2.3) & 24 \\
VI & $(68-77)$ & 4 \\
& $74 \cdot 3$ (SD 1.3) & \\
\hline
\end{tabular}

known, a mean anticipation of $8 \cdot 2$ (SD $10 \cdot 8$ ) (range -24 to +14 ) years was observed $(\mathrm{p}<0.05)$. Anticipation was more marked, although not significantly, in maternal $(10 \cdot 6$ ( SD $8 \cdot 6)$ years, $n=10)$ than in paternal $(6 \cdot 2$ (SD $12 \cdot 4$ ) years, $n=12$ ) transmissions. Evidence for anticipation in parent-offspring pairs remained statistically significant $(p<0.05)$, even if asymptomatic carriers were considered to have onset at the same age as the affected parent in order to minimise the bias introduced by the inability to observe late onset patients in the youngest generation.

A good correlation $(r=0.79)$ between the age at onset of symptoms and the number of repeats in the expanded allele was shown (fig 3 ), although there was wide variation among individual subjects. For example, age at onset varied from 25 to 55 years in patients with 72 CAG repeats. This result indicates that accurate predictions of age at onset cannot be made for unaffected mutation carriers.

Age at onset in women $(n=22)$ and men $(\mathrm{n}=24)$ did not diverge significantly (49.5 (SD $12 \cdot 4)$ and $41 \cdot 8$ (SD 13.3) years, $p>0 \cdot 05$ ). Furthermore, in five sibships (three pairs and two triplets) of different sexes, both the mean number of CAG repeats (73.0 (SD 3.5) and 73.2 (SD 1.8)) and the mean age at onset (34.8 (SD 9.5 ) and 38.8 (SD 10.9) years) were similar for men $(n=6)$ and women $(n=6)$. There was no difference in age at onset between these five mixed sibships and five sibships of the same sex $(8.6(\mathrm{SD} 6 \cdot 8)$ and $8.8(\mathrm{SD} 7 \cdot 8)$ years, respectively; $\mathrm{p}>0.05)$.

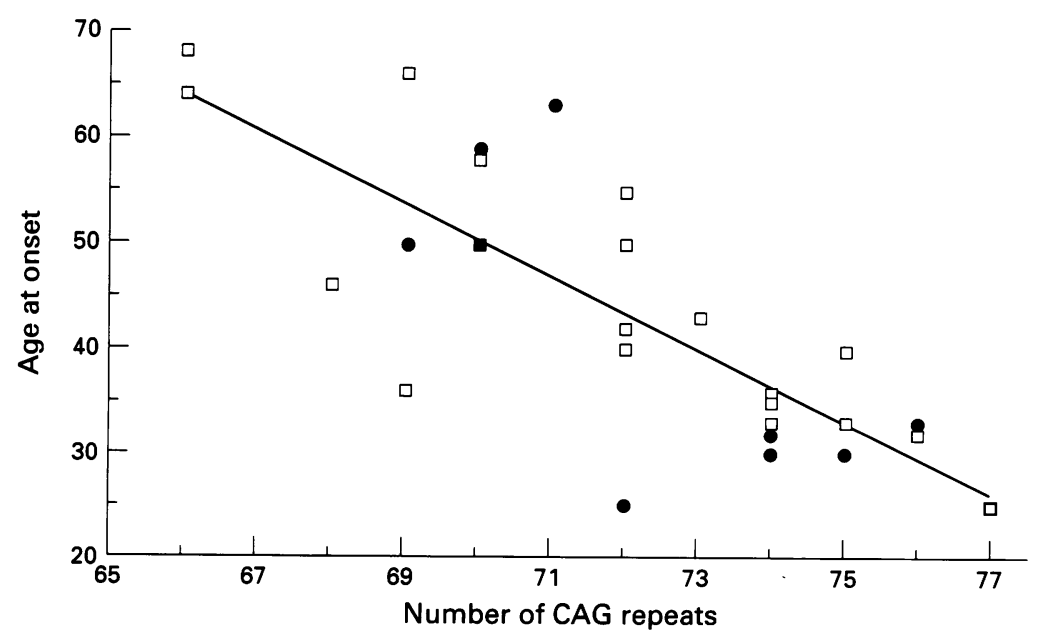

Figure 3 Correlation between age at onset (years) and the number of repeats of CAG in the mutated allele $(r=0 \cdot 79)$. Squares and hatched circles represent subjects who received the mutation from their father and their mother, respectively.
LINKAGE DISEQUILIBRIUM

Haplotypes segregating in the four families were reconstituted for four markers spanning $3 \mathrm{cM}$ on chromosome 14q: D14S291/D14S2802cM-AFM343vfl-lcM-D14S81. ${ }^{912}$ Markers D14S280 and AFM343vfl were determined as the centromeric and the telomeric markers, respectively. ${ }^{914}$ All patients and at risk carriers in the four families shared the same haplotype from D14S291 to D14S81 (D14S291: 1; D14S280: 5; AFM343vfl: 2; D14S81: 0). Furthermore, the alleles at D14S291 (0.26), AFM$343 \mathrm{vfl}(0 \cdot 22)$, and D14S81 (0.05) segregating with the disease were infrequent in the Brazilian population. Haplotype 1-5-2-0 was not present in 20 Brazilian and 60 French control chromosomes. Allele 5 at D14S280 also segregated with the disease in four French SCA3 families. ${ }^{91215}$ Although this allele is frequent in both French $(0.52)$ and Brazilian (0.58) populations, significant linkage disequilibrium was detected when data from both groups were combined $(p<0 \cdot 05)$.

\section{Discussion}

This study shows that the expansion of a CAG repeat sequence at the SCA3/MJD locus is involved in the pathogenesis of MJD in Brazilian families of Portuguese descent, as well as in Japanese kindreds.

By the analysis of 28 patients and 19 unaffected expansion carriers, we show that MJD shares the following features with four other neurodegenerative disorders, caused by expansions of CAG repeat ${ }^{16-21}$ : (1) distinct distributions of the number of repeats on normal and mutated alleles; (2) strong negative correlation between the number of repeats in the expanded allele and the age at onset of the disease; (3) instability of the expanded allele, particularly during paternal transmission; (4) evidence for anticipation in age at onset of the disease. Although the number of CAG repeats in the expanded allele in the Brazilian families was clearly different from the normal allele, was narrow in range (66-77), and negatively correlated with age at onset, it only accounted for $62 \%$ of the variance of this parameter, and therefore does not allow accurate prediction of the age at onset in asymptomatic gene carriers. The mutation was unstable in the four kindreds, since a mean increase in repeat number from generation to generation of 1.6 was observed, although when parent-child pairs were analysed, the repeat expanded $(n=7)$ or did not change $(n=4)$ or even decreased $(n=4)$ in individual cases. The mean increase, however, explains, at least in part, the mean anticipation of 13.3 years observed in successive generations and the mean anticipation of 8.2 years in 22 parent-offspring pairs.

In other disorders with unstable repeat expansions such as Hunting on's disease (HD), spinocerebellar ataxia 1 (SCA1), and dentatorubral and pallidoluysian atrophy (DRPLA), instability and tendency towards expansion was more marked in male than in female transmissions. ${ }^{1921-25}$ Maruyama et al $^{13}$ have recently reported greater instability of the 
CAG repeat in paternal transmissions, but they only observed expansions. Surprisingly, in the Brazilian families, larger increases, but also larger decreases, were transmitted by the fathers, which led to a mean increase of $0 \cdot 1$ repeat in father-child couples. This value is close to that obtained for maternal transmissions $(+0.4)$ for which the variance was smaller, although the number of transmissions was also smaller. Despite a greater paternal instability, no evidence of effect of paternal sex on the mean increase in CAG repeat numbers in parent-child couples could be found, because of the bidirectional instability during paternal transmission. The sex of the transmitting parent did not affect anticipation, which was in good agreement with the study of Barbeau et $a .^{26}$ This may be because of the absence of early onset cases ( $<20$ years) in this series, since large expansions, mostly of paternal origin, resulting in young onset in the children have been reported for HD, SCA1, and DRPLA. ${ }^{18192124}$ The gender of the patients also had no effect on repeat size or anticipation in contrast to the report of Kawakami et al. ${ }^{27}$

Finally, MJD/SCA3 segregated with the same haplotype in all the Brazilian kindreds studied, suggesting a common founder. The haplotype segregating with the disease carried allele 5 at the D14S280 locus in the four Portuguese-Brazilian families as well as in four French SCA3 linked kindreds with no known Portuguese ancestry. ${ }^{1215}$ Thus, since the same mutation is involved in MJD and SCA3 families, ${ }^{12 \mathrm{~A}}$ and linkage disequilibrium with D14S280 is significant, it is tempting to extend the founder effect to French families as well. However, different or recurrent mutations occurring in a predisposing haplotype could also account for the linkage disequilibrium as in HD. ${ }^{2829}$ Allele 5 of D14S280 was not associated with repeat lengths in the upper limit of the normal range in controls. This result does not support, but cannot rule out, the hypothesis of a predisposing haplotype for SCA3/MJD. Sasaki et $a l^{10}$ have observed linkage disequilibrium with D14S81 but not with D14S280 in 24 Japanese families, but it has not been proven that the MJD/SCA3 expansion is responsible for the disease in all of their families. Further studies in families of different ethnic origins with proven SCA3/MJD mutations are needed to answer this question definitively.

The authors are grateful to Drs Merle Ruberg and Josué Feingold for critical reading of the manuscript and to Alain Ploska and Drs Jean Weissenbach and Sophie Marc for their help. This study was supported by the Association Française contre les study was supported by the Association Française contre les
Myopathies, the Groupement de Recherches et d'Etudes sur les Génomes, The Verum Foundation, and the Association pour les Génomes, The Verum Foundation, and the Association pour le Développement de la Recherche
Neurologiques et Psychiatriques.

1 Rosenberg RN. Machado-Joseph disease: an autosomal dominant motor system degeneration. Mov Disord 1992 7:193-203

2 Séqueiros J, Coutinho P. Epidemiology and clinical aspects of Machado-Joseph disease. In: Harding AE, Deufel T, eds. Advances in neurology. Vol 61. Inherited ataxias. New York: Raven Press, 1993:139-53.

3 Takiyama Y, Ikemoto S, Tanaka Y, Mizuno Y, Yoshida M,
Yasuda N. A large Japanese family with Machado-Joseph disease: clinical and genetic studies. Acta Neurol Scand 1989;79:214-22.

4 Burt T, Blumbergs P, Currie B. A dominant hereditary ataxia resembling Machado-Joseph disease in Arnhem Land, Australia. Neurology 1993;43:1750-2.

5 Goldberg-Stern H, D'jaldetti R, Melamed E, Gadoth N. Machado-Joseph (Azorean) disease in a Yemenite Jewish family in Israel. Neurology 1994;44:1298-301.

6 Harding AE. Clinical features and classification of inherited ataxias. In: Harding AE, Deufel T, eds. Advances in neurlogy. Vol 61. Inherited ataxias. New York: Raven Press, 1993;1-14.

7 Takiyama Y, Nishizawa $\mathrm{M}$, Tanaka $\mathrm{H}$, et al. The gene for Machado-Joseph disease maps to human chromosome 14q. Nature Genet 1993;4:300-4.

8 Sequeiros J, Silveira I, Maciel P, et al. Genetic linkage studies of Machado-Joseph disease with chromosome 14q STRPs in 16 Portuguese-Azorean kindreds. Genomics 1994:21: 645-8.

9 Stevanin G, Sousa PS, Cancel G, et al. The gene for Machado-Joseph disease maps to the same $3 \mathrm{cM}$ interval as the spinal cerebellar ataxia 3 gene on chromosome 14q. Neurobiol Dis 1994;1:79-82.

10 Sasaki H, Wakisaka A, Takada A, et al. Mapping of the gene for Machado-Joseph disease within a $3.6 \mathrm{cM}$ interval gene for Machado-Joseph disease within a $3.6 \mathrm{cM}$ interval
flanked by D14S291/D14S280 and D 14S81, on the basis flanked by D14S291/D14S280 and D14S81, on the basis
of studies of linkage and linkage disequilibrium in 24 of studies of linkage and linkage disequilibrium in

11 Kawaguchi Y, Okamoto T, Taniwaki $M$, et al. CAG expansions in a novel gene for Machado-Joseph disease at chromosome 14q32.1. Nature Genet 1994;8:221-8.

12 Stevanin G, Cancel G, Dürr A, et al. The gene for spinal cerebellar ataxia 3 (SCA3) is located in a region of approximately $3 \mathrm{cM}$ on chromosome 14q24.3-q32.2. Am f Hum Genet 1995;56:193-201.

12A Cancel G, Abbas N, Stevanin G, et al. Marked phenotypic heterogeneity associated with expansion of a CAG repeat sequence at the spinocerebellar ataxia 3/Machado-Joseph disease locus. Am $\mathcal{F}$ Hum Genet (in press).

13 Maruyama H, Nakamura S, Matsuyama Z, et al. Molecular features of the CAG repeats and clinical manifestation of Machado-Joseph disease. Hum Molec Genet 1995;4: of Mach

14 Twist EC, Casaubon LK, Ruttledge MH, et al. MachadoJoseph disease maps to the same region of chromosome 14 as the spinocerebellar ataxia type 3 locus. $\mathcal{F}$ Med Genet
Jose 14 as the spinocer
$1995 ; 32: 25-31$.

15 Cancel G, Dürr A, Stevanin G, et al. Is DRPLA also linked to $14 \mathrm{q}$ ? Nature Genet 1994;6:8.

16 La Spada AR, Wilson EM, Lubahn DB, Harding AE, Fischbeck KH. Androgen receptor gene mutations in Xlinked spinal and bulbar muscular atrophy. Nature 1991; 352:77-9.

17 The Huntington's Disease Collaborative Research Group. A novel gene containing a trinucleotide repeat that is expanded and unstable on Huntington's disease chromosomes. Cell 1993;72:971-83.

18 Orr HT, Chung M-Y, Banfi S, et al. Expansion of an unstable trinucleotide CAG repeat in spinocerebellar ataxia type 1. Nature Genet 1993;4:221-6.

19 Nagafuchi S, Yanagisawa H, Sato K, et al. Dentatorubral and pallidoluysian atrophy expansion of an unstable CAG tri-

10 expression of the gene responsible for the triplet repeat disorder, dentatorubral and pallidoluysian atrophy disorder, dentatorubral and pallidoluys

21 Koide $\mathrm{R}$, Ikeuchi $\mathrm{T}$, Onodera $\mathrm{O}$, et al. Unstable expansion of CAG repeat in hereditary dentatorubral-pallidoluysian of CAG repeat in hereditary dentatorubral-pallido

22 Chung M-Y, Ranum LPW, Duvick LA, Servadio A, Zoghbi HY, Orr HT. Evidence for a mechanism predisposing to intergenerational CAG repeat instability in spinocerebellar ataxia type I. Nature Genet 1993;5:254-8.

23 Telenius H, Kremer HPH, Theilmann J, et al. Molecular analysis of juvenile Huntington disease: the major influence on $(\mathrm{CAG})_{\mathrm{n}}$ repeat is the sex of the affected parent. Hum Molec Genet 1993;2:1535-40.

24 Duyao M, Ambrose C, Myers R, et al. Trinucleotide repeat length instability and age of onset in Huntington's disease. Nature Genet 1993;4:387-92.

25 Matilla T, Volpini V, Genis D, et al. Presymptomatic analysis of spinocerebellar ataxia type 1 (SCA1) via the expansion of the SCA1 CAG-repeat in a large pedigree displaying anticipation and parental male bias. Hum Molec Genet anticipation and

26 Barbeau A, Roy M, Cunha L, et al. The natural history of Machado-Joseph disease: an analysis of 138 personally Machado-Joseph disease: an analysis of 138 pers

27 Kawakami H, Maruyama H, Nakamura S, et al. Unique features of the CAG repeats in Machado-Joseph disease. features of the CAG repeats
Nature Genet 1995;9:344-5.

28 Myers RH, MacDonald ME, Koroshetz WJ, et al. De novo expansion of a (CAG)n repeat in sporadic Huntington's disease. Nature Genet 1993;5:168-73.

29 Squitieri F, Andrew SE, Goldberg YP, et al. DNA haplotype analysis of Huntington disease reveals clues to the origins and mechanisms of CAG expansion and reasons for geographic variations of prevalence. Hum Molec Genet 1994; 3:2103-14. 